\title{
Pancreatic $\beta$-cell Imaging with High Affinity Peptide Ligands to the GLP-1 Receptor
}

\author{
Bikash Manandhar', William Silvers², Amit Kumar², Su-Tang Lo², \\ Xiankai Sun², and Jung-Mo Ahn ${ }^{1 *}$ \\ ${ }^{I}$ Dept. of Chemistry and Biochemistry, University of Texas at Dallas, Richardson, TX, 75080, USA; \\ ${ }^{2}$ Dept. of Radiology, University of Texas Southwestern Medical Center, Dallas, TX, 75390, USA
}

\section{Introduction}

In both type 1 and type 2 diabetes, early diagnosis and monitoring of the pancreatic beta-cell mass (BCM) change during the disease progression may be highly beneficial. As a specific beta-cell biomarker, glucagon-like peptide-1 receptor (GLP-1R) is found to be highly expressed in the pancreatic beta-cells and can serve as a suitable target for the development of molecular imaging probes [1]. Its natural ligand, glucagon-like peptide-1 (GLP-1), is an incretin and interacts with GLP$1 \mathrm{R}$ with nanomolar affinity, which makes it an outstanding candidate. There are several GLP-1 based PET imaging probes reported, but high renal uptake of these PET agents has been a concern for developing them for clinical use. As the beta-cells are dispersed throughout the pancreas and constitute less than $2 \%$ of the total mass, non-specific uptake by nearby tissues and organs like kidney and liver may decrease contrast of the signal dramatically. In our prior studies, we have demonstrated that strategically positioned lactam bridges at the $N$ - and $C$-terminus of GLP-1 can increase receptor affinity by stabilizing the helical structures in the peptide and enhance proteolytic stability. Based on the findings, we designed a bicyclic GLP-1 analog EM2198 having two lactam bridges between residues 18-22 and 30-34 [2,3]. D-Ala ${ }^{8}$ was also introduced to prevent the degradation by DPP-IV. The resulting bicyclic GLP-1 analog EM2198 was found to be remarkably stable, and a PET imaging agent for the pancreatic $\beta$-cells was developed by conjugating to DOTA and labeling with ${ }^{64} \mathrm{Cu}[4]$.

\section{Results and Discussion}

To lower non-specific uptake by nearby organs, we have modified our previously reported bicyclic GLP-1 analog EM2198 (1), by introducing hydrophilic tails, such as penta-glutamic acids (2), pentaserines (3) and penta-lysines (4). These modifications were found to make no change in receptor activation capability (Figure 1). These peptides were examined for their utility as PET agents for imaging the $\beta$-cells. PET/CT imaging probes were constructed by conjugating to NOTA. After labeling with Ga-68, static PET/CT scans were acquired on a Siemens Inveon PET/CT multimodality system using normal C57BL/6 mice.

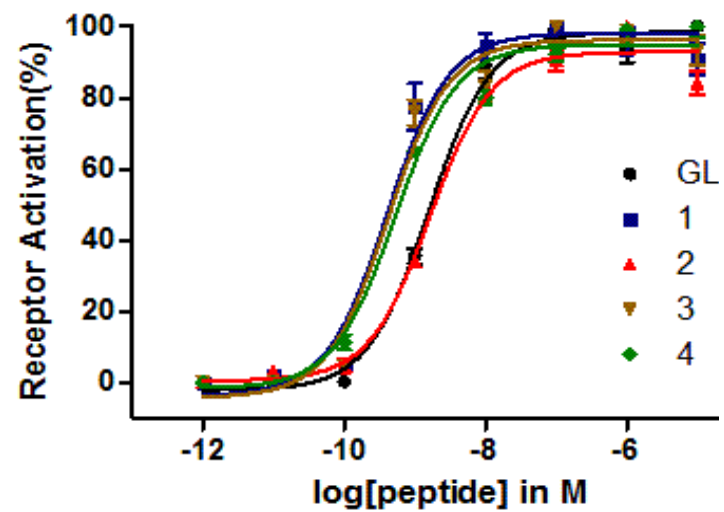

Fig. 1. GLP-1R activation by the GLP-1 analogs with hydrophilic tails.
PET tracers derived from these peptides were examined for their capability of enhancing the specific pancreatic uptake and lowering non-specific adsorption to nearby organs. Increase in polarity by introducing the hydrophilic tails appears to enhance imaging quality presumably by decreasing plasma protein binding and diminishing non-specific uptake by other organs like liver and lungs. The pancreas to kidney uptake ratios (Table 1) of the modified peptides 2-4 were found to be comparable to the parent peptide $\mathbf{1}$. The increased hydrophilicity may have slightly promoted clearance via renal route. The peptide bearing hydroxyl groups $\mathbf{3}$ appeared to be marginally superior to the other peptides with charged tails 2 and $\mathbf{4}$ (Figure 2). 
Table 1. Specific uptake ratios of GLP-1 based PET tracers.

\begin{tabular}{ccccc}
\hline Uptake ratio & \multicolumn{4}{c}{ GLP-1 analogs } \\
& 1 & 2 & 3 & 4 \\
\hline Pancreas/Liver & 0.76 & 1.06 & 1.19 & 1.21 \\
Pancreas/Lungs & 0.60 & 1.15 & 1.37 & 0.86 \\
Pancreas/Kidney & 0.05 & 0.02 & 0.04 & 0.02 \\
\hline
\end{tabular}

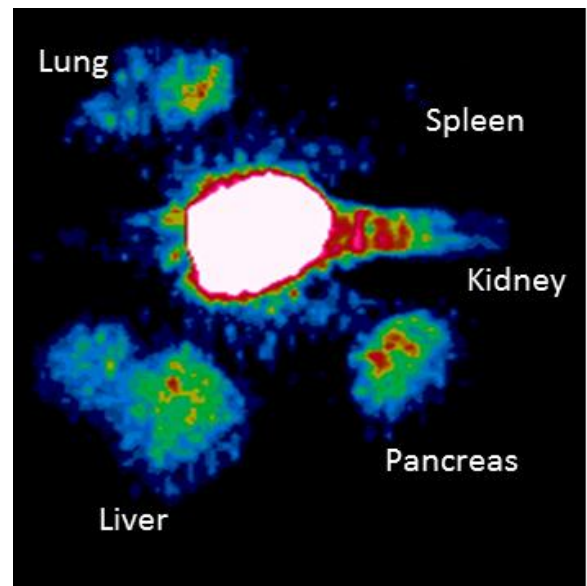

Fig. 2. Ex vivo PET image of peptide 3 in normal mice.

\section{Acknowledgments}

This work was supported in part by the Welch Foundation (AT-1595), the Juvenile Diabetes Research Foundation (37-2011-20, 37-2011-632), and National Institutes of Health (R01 DK092163-01).

\section{References}

1. Manandhar, B., Ahn, J.-M. J. Med. Chem. 58, 1020-1037 (2015), http://dx.doi.org/doi:10.1021/jm500810s

2. Murage, E.N., et al. Bioorg. Med. Chem. 16, 10106-10112 (2008),

http://dx.doi.org/doi:10.1016/j.bmc.2008.10.006

3. Murage, E.N., et al. J. Med. Chem. 53, 6412-6420 (2010), http://dx.doi.org/doi:10.1021/jm100602m

4. Manandhar, B., et al., in Lebl, M. (Ed.) Peptides: Peptides Across the Pacific (Proceedings of the 23rd American Peptide Symposium), American Peptide Society, San Diego, 2013, p. 172, http://dx.doi.org/10.17952/23APS.2013.172 